Punjab University Journal of Mathematics (2021),53(12),861-879

https://doi.org/10.52280/pujm.2021.531203

\title{
Some new generalizations for exponentially $(s, m)$-preinvex functions considering generalized fractional integral operators
}

\author{
Farhat Safdar \\ Department of Mathematics, \\ SBK Women's University Quetta, Pakistan, \\ Email: farhat900@yahoo.com \\ Muhammad Attique \\ Department of Mathematics, \\ University of Agriculture Faisalabadta, Pakistan, \\ Email: muhammad.attique@uaf.edu.pk
}

Received: 10 June, 2021 / Accepted: 23 September, 2021 / Published online: 24 December, 2021

\begin{abstract}
The generalized fractional integral has been one of the most useful operators for modelling non-local behaviors by fractional differential equations. It is considered, for several integral inequalities by introducing the concept of exponentially $(s, m)$-preinvexity. These variants derived via an extended Mittag-Leffler function based on boundedness, continuity and Hermite-Hadamard type inequalities. The consequences associated with fractional integral operators are more general and also present the results for convexity theory. Moreover, we point out that the variants are useful in solving the problems of science, engineering and technology where the Mittag-Leffler function occurs naturally.
\end{abstract}

\begin{abstract}
AMS (MOS) Subject Classification Codes: 35S29; 40S70; 25 U09
Key Words: Exponentially $(s, m)$-preinvex functions, Integral inequalities, Mittag-Leffler functions, preinvex functions, convex function..
\end{abstract}

\section{INTRODUCTION}

The imperative study of derivatives and integrals has been done in calculus. The fractional calculus is evolved when he classical derivative was convoluted with the strength regulation kind kernel. For applications in bioengineering, financial economics, and pure mathematics, we refer the interested readers $[1,2]$. The information in the context of fractional operators about rheological models can be investigated in $[3,4,51,52,53,54$, $55,56,57,58]$. In perspective on the broad utilization of such frameworks, numerous scientists went to the examination of the hypothetical parts of fractional differential conditions. Specifically, there was unique consideration regarding demonstrating the integral inequalities for fractional systems enhanced with an assortment of classical and non-classical 
(nonlocal) operators with the guide of advance strategies for functional investigation, see $[5,6,7,8,9,10,11,12,13,14,15,16,17,18,59,60,61,62,63,64,65]$. Fractional integral inequalities involving convex functions played a significant role in the mathematical analysis due to their prominent features and convenient characterizations. Integral inequalities have been extensively studied in the literature and their variant forms presented by fractional integrals operators are Hermite-Hadamard, Ostrowski, Lyenger and so forth. The most captivating inequality is the Hermite-Hadamard inequality, which can be used to find lower and upper bounds for fractional integral for some given convex functions.

The following well-known inequality

$$
\hbar\left(\frac{x_{1}+x_{2}}{2}\right) \leq \frac{1}{x_{2}-x_{1}} \int_{x_{1}}^{x_{2}} \hbar(z) d z \leq \frac{\hbar\left(x_{1}\right)+\hbar\left(x_{2}\right)}{2}
$$

holds for convex functions and is known as the Hermite-Hadamard inequality. This inequality has for quite some time been known as Hadamard's inequality. This inequality of Hermite was found by Mitrinovic, who he had discovered sooner than Hadamard and is referred to now in the writing as Hermite-Hadamard's inequality. The significance of this inequality is because of the way that it is identical to the meaning of convexity under specific conditions. The inequality (1. 1 ) has been contemplated by a few analysts because of its potential applications, see [19, 20, 21, 22, 23, 24, 25, 26, 27, 28].

Now we demonstrate some basic preliminaries concerning to convexity and preinvexity as follows:

\section{NotATIONS AND PRELIMINARIES}

Now we demonstrate some basic preliminaries concerning to convexity and preinvexity as follows:

Definition 2.1. A mapping $\hbar: \mathcal{K} \rightarrow \mathcal{R}$ is said to be convex, if the inequality

$$
\left.\hbar\left(\zeta x_{1}+(1-\zeta) x_{2}\right)\right) \leq \zeta \hbar\left(x_{1}\right)+(1-\zeta) \hbar\left(x_{2}\right)
$$

holds for all $x_{1}, x_{2} \in \mathcal{K}$ and $\zeta \in[0,1]$.

Awan et al. [29] gave the concept of exponentially convex function.

Definition 2.2. A real-valued mapping $\hbar: \mathcal{K} \rightarrow \mathcal{R}$ is said to be exponentially convex function if the inequality

$$
\left.\hbar\left(\zeta x_{1}+(1-\zeta) x_{2}\right)\right) \leq \zeta \frac{\hbar\left(x_{1}\right)}{e^{\vartheta x_{1}}}+(1-\zeta) \frac{\hbar\left(x_{2}\right)}{e^{\vartheta x_{2}}}
$$

holds for all $x_{1}, x_{2} \in \mathcal{K}, \zeta \in[0,1]$ and $\vartheta \in \mathcal{R}$

Exponentially convex functions are introduced by Bernstein [30] in covariance formation then Avriel [31] expounded the concept of exponentially convex function in linear programming. Alirezaei and Mathar [32] explored the application of exponentially convex 
functions in information theory as well in statistical theory. For more details and information, see $[33,34]$.

In [35], Craven expounded the term due to their properties discussed as invariance by convexity. In [36], Hanson outfitted the possibility of differentiable invex functions in the context of their accurate global optimum behavior and obtained the significance generalization of convex functions is that of invex functions. Weir and Mond [37] explored the generalizations of convex functions is that of preinvex functions and utilized it to buildup the duality and the sufficient optimality conditions in nonlinear programming. In [38], Noor introduced another version of Hermite-Hadamard inequality for preinvex functions. Mohan and Neogy [39] explored a famous result, which is known as Condition C. Mititelu [40] investigated well-known concept of invex sets as follows:

Now, we will give the definition of preinvex functions with some basic properties. Suppose that $\Omega \subset \mathcal{R}$ be a set, $\hbar: \Omega \rightarrow \mathcal{R}$ be a continuous function and $\eta(.,):. \Omega \times \Omega \rightarrow \mathcal{R}$ be a continuous bifunction.

Definition 2.3. [40]A set $\Omega \subset \mathcal{R}$ is said to be invex set $w$. $r$. to the bifunction $\eta(.,$.$) , if and$ only if

$$
x_{1}+\zeta \eta\left(x_{2}, x_{1}\right) \in \Omega, \quad \forall x_{1}, x_{2} \in \Omega, \zeta \in[0,1] .
$$

It is also called $\eta$-connected set. Note that, if $\eta\left(x_{2}, x_{1}\right)=x_{2}-x_{1}$, this implies that every convex set is an invex set, but the converse is not true. We will use the set $\Omega$ as an invex set, unless otherwise it is specified.

Definition 2.4. [37] A mapping $\hbar$ on the invex set $\Omega$ is said to be preinvex function $w$. $r$. to $\eta(.,$.$) , if the inequality$

$$
\hbar\left(x_{1}+\zeta \eta\left(x_{2}, x_{1}\right)\right) \leq(1-\zeta) \hbar\left(x_{1}\right)+\zeta \hbar\left(x_{2}\right)
$$

holds $\forall x_{1}, x_{1}+\eta\left(x_{2}, x_{1}\right) \in \Omega$ and $\zeta \in[0,1]$.

We now introduce the concept of exponentially preinvex, exponentially $s$-preinvex and exponentially $m$-preinvex function, respectively.

Definition 2.5. A real-valued mapping $\hbar$ on the invex set $\Omega$ is said to be exponentially preinvex w. r. to $\eta(.,$.$) , if the inequality$

$$
\hbar\left(x_{1}+\zeta \eta\left(x_{2}, x_{1}\right)\right) \leq(1-\zeta) \frac{\hbar\left(x_{1}\right)}{e^{\vartheta x_{1}}}+\zeta \frac{\hbar\left(x_{2}\right)}{e^{\vartheta x_{2}}}
$$

holds for all $x_{1}, x_{1}+\eta\left(x_{2}, x_{1}\right) \in \Omega, \zeta \in[0,1]$, and $\vartheta \in \mathcal{R}$.

Definition 2.6. Let $s \in(0,1]$ and a real-valued mapping $\hbar$ on the invex set $\Omega$ is said to be exponentially s-preinvex w. r. to $\eta(.,$.$) , if the inequality$

$$
\hbar\left(x_{1}+\zeta \eta\left(x_{2}, x_{1}\right)\right) \leq(1-\zeta)^{s} \frac{\hbar\left(x_{1}\right)}{e^{\vartheta x_{1}}}+\zeta^{s} \frac{\hbar\left(x_{2}\right)}{e^{\vartheta x_{2}}}
$$

holds for all $x_{1}, x_{1}+\eta\left(x_{2}, x_{1}\right) \in \Omega, \zeta \in[0,1]$, and $\vartheta \in \mathcal{R}$.

Now we extend the concept of exponentially $m$-preinvexity, by some transformation we will get $m$-preinvex functions and $m$-convex functions introduced by Toader [41]. 
Definition 2.7. A real-valued mapping $\hbar$ on the invex set $\Omega$ is said to be exponentially m-preinvex w. r. to $\eta(.,$.$) , if the inequality$

$$
\hbar\left(x_{1}+m \zeta \eta\left(x_{2}, x_{1}\right)\right) \leq(1-\zeta)^{s} \frac{\hbar\left(x_{1}\right)}{e^{\vartheta x_{1}}}+m \zeta^{s} \frac{\hbar\left(x_{2}\right)}{e^{\vartheta x_{2}}}
$$

holds for all $x_{1}, x_{1}+\eta\left(x_{2}, x_{1}\right) \in[0, b], \zeta \in[0,1], m \in[0,1]$ and $\vartheta \in \mathcal{R}$.

In 1903, Gosta [42] introduced the idea of Mittag-Leffler function $E_{\sigma}(\zeta)$ as

$$
E_{\sigma}(\zeta)=\sum_{n=0}^{\infty} \frac{\zeta^{n}}{\Gamma(\sigma n+1)},
$$

where $\zeta, \sigma \in \mathbb{C}, \Re(\sigma)$ and $\Gamma($.$) is the gamma function.$

If $\sigma=1$ then we obtain the direct generalization of the exponential function is MittagLeffler function. In the arrangement of fractional differential equations and integral equations, the Mittag-Leffler work emerges normally. Due to its significance Mittag-Leffler work is additionally summed up and stretched out by numerous specialists, we allude the peruser to [5, 6, 33, 43, 44, 45, 47, 48]. As of late in [43], Andric et al. presented generalized Mittag-Leffler work defined as pursues:

Definition 2.8. [43] Let $\omega, \mu, \sigma, \jmath, \gamma, \nu \in \mathbb{C}, \Re(\mu), \Re(\sigma), \Re(\jmath), \Re(\nu), \Re(\gamma)>0$ with $q \geq$ $0, \delta>0$ and $0<\kappa \leq \delta+\Re(\mu)$. Let $\left.\hbar \in L_{[} x_{1}, x_{2}\right]$ and $z \in\left[x_{1}, x_{2}\right]$. Then the generalized fractional integral operators having Mittag-Leffler function are defined by:

$$
\left(\epsilon_{\mu, \sigma, \jmath, \omega, x_{1}^{+}} \hbar\right)(z ; q)=\int_{x_{1}}^{z} E_{\mu, \sigma, \jmath}^{\gamma, \delta, \nu}\left(\omega(z-\zeta)^{\mu} ; q\right) \hbar(\zeta) d \zeta
$$

and

$$
\left(\epsilon_{\mu, \sigma, \jmath, \omega, x_{2}^{-}} \hbar\right)(z ; q)=\int_{z}^{x_{2}} E_{\mu, \sigma, j}^{\gamma, \delta, \kappa, \nu}\left(\omega(\zeta-z)^{\mu} ; q\right) \hbar(\zeta) d \zeta
$$

where $E_{\mu, \sigma, j}^{\gamma, \delta, \kappa, \nu}($.$) is the Mittag-Leffler function defined as:$

$$
E_{\mu, \sigma, j}^{\gamma, \delta, \kappa, \nu}(\zeta ; q)=\sum_{n=0}^{\infty} \frac{\beta_{q}(\gamma+n \kappa, \nu-\gamma)}{\beta(\gamma, \nu-\gamma)} \frac{(\nu)_{n \kappa} \zeta^{n}}{\Gamma(\mu n+\sigma)(\jmath)_{n \delta}},
$$

and $\beta_{q}$ is the generalized beta function defined as follows:

$$
\beta_{q}(x, y)=\int_{0}^{1} \zeta^{x-1}(1-\zeta)^{y-1} e^{-\frac{q}{\zeta(1-\zeta)}} d \zeta
$$

and $(\nu)_{n \kappa}$ is the Pochhammer symbol defined by $(\nu)_{n \kappa}=\frac{\Gamma(\nu+n \kappa)}{\Gamma(\nu)}$.

Remark 2.9. Definition 2.8 is the generalization of the following fractional integral operators containing Mittag-Leffler function:

(1) choosing $q=0$, we have the fractional integral operators defined by Salim-Faraj in [33].

(2) choosing $\jmath=\delta=1$, we have the fractional integral operators defined by Rahman et 
al. in [6].

(3) choosing $q=0$ and $\jmath=\delta=1$, we have the fractional integral operators defined by Srivastava-Tomovski in [48].

(4) choosing $q=0$ and $\jmath=\delta=\kappa=1$, we have the fractional integral operators defined by Prabhakar in [5].

(5) choosing $q=\omega=0$, we have the right-sided and left-sided Riemann-Liouville fractional integrals.

Farid et al. [18] obtained as follows:

$$
\left(\epsilon_{\mu, \sigma, \jmath, \omega, x_{1}^{+}}^{\gamma, \delta, \kappa, \nu}\right)(z ; q)=\left(z-x_{1}\right)^{\sigma} E_{\mu, \sigma+1, j}^{\gamma, \delta, \kappa, \nu}\left(\omega\left(z-x_{1}\right)^{\mu} ; q\right)
$$

and

$$
\left(\epsilon_{\mu, \tau, j, \omega, x_{2}^{-}}^{\gamma, \delta, \kappa, \nu} 1\right)(z ; q)=\left(x_{2}-z\right)^{\tau} E_{\mu, \tau+1, j}^{\gamma, \delta, \kappa, \nu}\left(\omega\left(x_{2}-z\right)^{\mu} ; q\right) .
$$

The principal purpose of this paper is to introduce the concept of exponentially $(s, m)$ preinvex function in section 3 , which is the generalizations of $s$-preinvex, $m$-preinvex, exponentially preinvex, exponentially $s$-preinvex and exponentially $m$-preinvex functions, and explore the estimates of concerning to the Mittag-Leffler functions in their kernels. Our outcomes provide several new generalizations by some variations in parameters.

\section{MAIn RESUlts}

In this section, we introduced the concept of exponentially $(s, m)$-preinvex functions, which is the main motivation of this paper.

Definition 3.1. Let $s \in(0,1]$ and a real valued mapping on invex set $\Omega$ is said to be exponentially $(s, m)$-preinvex, if the inequality

$$
\hbar\left(x_{1}+\zeta \eta\left(m x_{2}, x_{1}\right)\right) \leq(1-\zeta)^{s} \frac{\hbar\left(x_{1}\right)}{e^{\vartheta x_{1}}}+m \zeta^{s} \frac{\hbar\left(x_{2}\right)}{e^{\vartheta x_{2}}}
$$

holds for all $x_{1}, x_{1}+\eta\left(x_{2}, x_{1}\right) \in \Omega, \zeta \in[0,1], m \in[0,1]$ and $\vartheta \in \mathcal{R}$.

Remark 3.2. In Definition 3.1:

(1) choosing $m=1$, we attain exponentially s-preinvex functions.

(2) choosing $\vartheta=0$, we attain $(s, m)$-preinvex functions.

(3) choosing $\vartheta=0, m=1$, we attain s-preinvex functions.

(4) choosing $\vartheta=0, s=1, m=1$, we attain classical preinvex functions.

(5) choosing $\vartheta=0, s=1, m=1$ with $\eta\left(x_{2}, x_{1}\right)=x_{2}-x_{1}$, we attain classical convex functions.

Theorem 3.3. Suppose a real valued function $\hbar:\left[x_{1}, x_{1}+\eta\left(x_{2}, x_{1}\right)\right] \rightarrow \mathcal{R}$ be an exponentially $(s, m)$-preinvex function, then the following fractional integral inequality for 
(2. 9 ) and (2. 10$)$ holds:

$$
\begin{aligned}
& \left(\epsilon_{\mu, \sigma, j, \omega, x_{1}^{+}}^{\gamma, \delta, \kappa, \nu} \hbar\right)(z ; q)+\left(\epsilon_{\mu, \tau, j, \omega,\left(x_{1}+\eta\left(x_{2}, x_{1}\right)^{-}\right)}^{\gamma, \delta, \kappa, \nu} \hbar\right)(z ; q) \\
& \leq \frac{\left(\zeta-x_{1}\right)\left(\epsilon_{\mu, \sigma-1, j, x_{1}^{+}}^{\gamma, \delta, \kappa, \nu} 1\right)(z ; q)}{s+1}\left(\frac{\hbar\left(x_{1}\right)}{e^{\vartheta_{1} x_{1}}}+m \frac{\hbar(z)}{e^{\vartheta_{1} z}}\right) \\
& +\frac{\left(x_{1}+\eta\left(x_{2}, x_{1}\right)-z\right)\left(\epsilon_{\mu, \tau-1, j,\left(x_{1}+\eta\left(x_{2}, x_{1}\right)\right)^{\gamma}}^{\gamma, \delta, \kappa)}\right)(z ; q)}{s+1} \\
& \times\left(\frac{\hbar\left(x_{1}+\eta\left(x_{2}, x_{1}\right)\right)}{e^{\vartheta_{2}\left(x_{1}+\eta\left(x_{2}, x_{1}\right)\right)}}+m \frac{\hbar(z)}{e^{\vartheta_{2} z}}\right),
\end{aligned}
$$

for all $z \in\left[x_{1}, x_{1}+\eta\left(x_{2}, x_{1}\right)\right]$.

Proof. Let $z \in\left[x_{1}, x_{1}+\eta\left(x_{2}, x_{1}\right)\right]$. Then for $\zeta \in\left[x_{1}, z\right)$ and $\sigma \geq 1$, we have the following inequality:

$$
\begin{array}{r}
(z-\zeta)^{\sigma-1} E_{\mu, \sigma, j}^{\gamma, \delta, \kappa, \nu}\left(\omega(z-\zeta)^{\mu} ; q\right) \\
\leq\left(z-x_{1}\right)^{\sigma-1} E_{\mu, \sigma, j}^{\gamma, \delta, \kappa, \nu}\left(\omega\left(z-x_{1}\right)^{\mu} ; q\right) .
\end{array}
$$

Since $\hbar$ is exponentially $(s, m)$-preinvex, we have

$$
\hbar(\zeta) \leq\left(\frac{z-\zeta}{z-x_{1}}\right)^{s} \frac{\hbar\left(x_{1}\right)}{e^{\vartheta_{1} x_{1}}}+m\left(\frac{\zeta-x_{1}}{z-x_{1}}\right)^{s} \frac{\hbar(z)}{e^{\vartheta_{1} z}} .
$$

Taking product (3. 14 ) and (3. 15 ) and then integrating with respect to $\zeta$ from $x_{1}$ to $z$, we have

$$
\begin{aligned}
& \int_{x_{1}}^{z}(z-\zeta)^{\sigma-1} E_{\mu, \sigma, j}^{\gamma, \delta, \nu}\left(\omega(z-\zeta)^{\mu} ; q\right) \hbar(\zeta) d \zeta \\
& \leq \int_{x_{1}}^{z}\left(z-x_{1}\right)^{\sigma-1} E_{\mu, \sigma, \delta, \nu}^{\gamma, \delta, \nu}\left(\omega\left(z-x_{1}\right)^{\mu} ; q\right) \\
& \quad \times\left[\left(\frac{z-\zeta}{z-x_{1}}\right)^{s} \frac{\hbar\left(x_{1}\right)}{e^{\vartheta_{1} x_{1}}}+m\left(\frac{\zeta-x_{1}}{z-x_{1}}\right)^{s} \frac{\hbar(z)}{e^{\vartheta_{1} z}}\right] d \zeta .
\end{aligned}
$$

Using Definition 2.8, we have

$$
\begin{aligned}
& \left(\epsilon_{\mu, \sigma, j, \omega, x_{1}^{+}}^{\gamma, \delta, \kappa, \nu} \hbar\right)(z ; q) \\
& \leq \frac{\left(z-x_{1}\right)\left(\epsilon_{\mu, \sigma-1, \jmath, x_{1}^{+}}^{\gamma, \delta, \kappa,} 1\right)(z ; q)}{s+1}\left(\frac{\hbar\left(x_{1}\right)}{e^{\vartheta_{1} x_{1}}}+m \frac{\hbar(z)}{e^{\vartheta_{1} z}}\right) .
\end{aligned}
$$


Analogously, for $\zeta \in\left(z, x_{1}+\eta\left(x_{2}, x_{1}\right)\right]$ and $\tau \geq 1$, we have

$$
\begin{aligned}
& (\zeta-z)^{\tau-1} E_{\mu, \tau, \jmath}^{\gamma, \delta, \kappa, \nu}\left(\omega(\zeta-z)^{\mu} ; q\right) \\
& \leq\left(x_{1}+\eta\left(x_{2}, x_{1}\right)-z\right)^{\tau-1} \\
& \times E_{\mu, \tau, j}^{\gamma, \delta, \kappa}\left(\omega\left(x_{1}+\eta\left(x_{2}, x_{1}\right)-z\right)^{\mu} ; q\right) .
\end{aligned}
$$

Further, from exponentially $(s, m)$-convexity of $\hbar$, we have

$$
\begin{aligned}
& \hbar(\zeta) \leq\left(\frac{\zeta-z}{x_{1}+\eta\left(x_{2}, x_{1}\right)-z}\right)^{s} \frac{\hbar\left(x_{1}+\eta\left(x_{2}, x_{1}\right)\right)}{e^{\vartheta_{2}\left(x_{1}+\eta\left(x_{2}, x_{1}\right)\right)}} \\
& +m\left(\frac{x_{1}+\eta\left(x_{2}, x_{1}\right)-\zeta}{x_{1}+\eta\left(x_{2}, x_{1}\right)-z}\right)^{s} \frac{\hbar(z)}{e^{\vartheta_{2} z}}
\end{aligned}
$$

Taking product (3.18) and (3.19) and then integrating with respect to $\zeta$ from $z$ to $x_{1}+$ $\eta\left(x_{2}, x_{1}\right)$, we have

$$
\begin{aligned}
& \int_{z}^{x_{1}+\eta\left(x_{2}, x_{1}\right)}(\zeta-z)^{\tau-1} E_{\mu, \tau, j}^{\gamma, \delta, \kappa, \nu}\left(\omega(\zeta-z)^{\mu} ; q\right) \hbar(\zeta) d \zeta \\
& \leq \int_{z}^{x_{1}+\eta\left(x_{2}, x_{1}\right)}\left(x_{1}+\eta\left(x_{2}, x_{1}\right)-z\right)^{\tau-1} \\
& \quad \times E_{\mu, \tau, j}^{\gamma, \delta, \kappa, \nu}\left(\omega\left(x_{1}+\eta\left(x_{2}, x_{1}\right)-z\right)^{\mu} ; q\right) \\
& \quad \times \quad\left[\left(\frac{\zeta-z}{x_{1}+\eta\left(x_{2}, x_{1}\right)-z}\right)^{s} \frac{\hbar\left(x_{1}+\eta\left(x_{2}, x_{1}\right)\right)}{e^{\vartheta_{2}\left(x_{1}+\eta\left(x_{2}, x_{1}\right)\right)}}\right. \\
& \left.\quad+m\left(\frac{x_{1}+\eta\left(x_{2}, x_{1}\right)-\zeta}{x_{1}+\eta\left(x_{2}, x_{1}\right)-z}\right)^{s} \frac{\hbar(z)}{e^{\vartheta_{2} z}}\right] d \zeta .
\end{aligned}
$$

Again, using Definition 2.8, we get

$$
\begin{aligned}
& \left(\epsilon_{\mu, \tau, j, \omega,\left(x_{1}+\eta\left(x_{2}, x_{1}\right)\right)^{-}}^{\gamma, \delta, \kappa, \nu}\right)(z ; q) \\
& \leq \frac{\left(x_{1}+\eta\left(x_{2}, x_{1}\right)-z\right)\left(\epsilon_{\mu, \tau-1, \jmath,\left(x_{1}+\eta\left(x_{2}, x_{1}\right)\right)^{-}}^{\gamma, \delta, \kappa, \nu}\right)(z ; q)}{s+1} \\
& \quad \times\left(\frac{\hbar\left(x_{1}+\eta\left(x_{2}, x_{1}\right)\right)}{e^{\vartheta_{2}\left(x_{1}+\eta\left(x_{2}, x_{1}\right)\right)}}+m \frac{\hbar(z)}{e^{\vartheta_{2} z}}\right) .
\end{aligned}
$$

Summing up (3.18) and (3.21), we deduce the inequality (3.13).

Corollary 3.4. If $\hbar \in L_{\infty}\left[x_{1}, x_{1}+\eta\left(x_{2}, x_{1}\right)\right]$, then under the assumption of Theorem 3.3, we have

$$
\begin{aligned}
& \left(\epsilon_{\mu, \sigma, j, \omega, x_{1}^{+}}^{\gamma, \delta} \hbar\right)(z ; q)+\left(\epsilon_{\mu, \tau, \jmath, \omega,\left(x_{1}+\eta\left(x_{2}, x_{1}\right)^{-}\right)}^{\gamma, \delta, \kappa, \nu}\right)(z ; q) \\
& \leq \frac{\|\hbar\|_{\infty}}{s+1}\left[\left(\zeta-x_{1}\right)\left(\epsilon_{\mu, \sigma-1, \jmath, x_{1}^{+}}^{\gamma, \delta, \kappa, \nu}\right)(z ; q)\left(\frac{1}{e^{\vartheta_{1} x_{1}}}+m \frac{1}{e^{\vartheta_{1} z}}\right)\right. \\
& \quad+\left(x_{1}+\eta\left(x_{2}, x_{1}\right)-z\right)\left(\epsilon_{\mu, \tau-1, j,\left(x_{1}+\eta\left(x_{2}, x_{1}\right)\right)^{-}}^{\gamma, \delta, \kappa, \nu}\right)(z ; q) \\
& \left.\quad \times\left(\frac{1}{e^{\vartheta_{2}\left(x_{1}+\eta\left(x_{2}, x_{1}\right)\right)}}+m \frac{1}{e^{\vartheta_{2} z}}\right)\right] .
\end{aligned}
$$


Corollary 3.5. Choosing $m=1$ and $\hbar \in L_{\infty}\left[x_{1}, x_{1}+\eta\left(x_{2}, x_{1}\right)\right]$, then under the assumption of Theorem 3.3, we have

$$
\begin{aligned}
& \left(\epsilon_{\mu, \sigma, \jmath, \omega, x_{1}^{+}}^{\gamma, \delta, \kappa, \nu}\right)(z ; q)+\left(\epsilon_{\mu, \tau, \jmath, \omega,\left(x_{1}+\eta\left(x_{2}, x_{1}\right)^{-}\right)}^{\gamma, \delta, \kappa, \nu}\right)(z ; q) \\
& \leq \frac{\|\hbar\|_{\infty}}{s+1}\left[\left(\zeta-x_{1}\right)\left(\epsilon_{\mu, \sigma-1, \jmath, x_{1}^{+}}^{\gamma, \delta, \kappa, \nu}\right)(z ; q)\left(\frac{1}{e^{\vartheta_{1} x_{1}}}+\frac{1}{e^{\vartheta} \gamma_{1}}\right)\right. \\
& \quad+\left(x_{1}+\eta\left(x_{2}, x_{1}\right)-z\right)\left(\epsilon_{\mu, \tau-1, \jmath,\left(x_{1}+\eta\left(x_{2}, x_{1}\right)\right)^{\gamma}}^{\gamma, \delta, \kappa, \nu}\right)(z ; q) \\
& \left.\quad \times\left(\frac{1}{e^{\vartheta_{2}\left(x_{1}+\eta\left(x_{2}, x_{1}\right)\right)}}+\frac{1}{e^{\vartheta_{2} z}}\right)\right] .
\end{aligned}
$$

Corollary 3.6. Choosing $m=s=1$ and $\hbar \in L_{\infty}\left[x_{1}, x_{1}+\eta\left(x_{2}, x_{1}\right)\right]$, then under the assumption of Theorem 3.3, we have

$$
\begin{aligned}
& \left(\epsilon_{\begin{array}{l}
\gamma, \delta, \kappa, \nu \\
\mu, \sigma, \omega, x_{1}^{+}
\end{array}}^{\gamma} \hbar\right)(z ; q)+\left(\epsilon_{\mu, \tau, \jmath, \omega,\left(x_{1}+\eta\left(x_{2}, x_{1}\right)^{-}\right)}^{\gamma, \delta, \kappa, \nu}\right)(z ; q) \\
& \leq \frac{\|\hbar\|_{\infty}}{2}\left[\left(\zeta-x_{1}\right)\left(\epsilon_{\mu, \sigma-1, \jmath, x_{1}^{+}}^{\gamma, \delta, \kappa, \nu}\right)(z ; q)\left(\frac{1}{e^{\vartheta_{1} x_{1}}}+\frac{1}{e^{\vartheta_{1} z}}\right)\right. \\
& \quad+\left(x_{1}+\eta\left(x_{2}, x_{1}\right)-z\right)\left(\epsilon_{\mu, \tau-1, \jmath,\left(x_{1}+\eta\left(x_{2}, x_{1}\right)\right)^{\gamma}}^{\gamma, \delta, \kappa, \nu}\right)(z ; q) \\
& \left.\quad \times\left(\frac{1}{e^{\vartheta_{2}\left(x_{1}+\eta\left(x_{2}, x_{1}\right)\right)}}+\frac{1}{e^{\vartheta_{2} z}}\right)\right] .
\end{aligned}
$$

Corollary 3.7. Choosing $\eta\left(x_{2}, x_{1}\right)=x_{2}-x_{1}$ and $\hbar \in L_{\infty}\left[x_{1}, x_{2}\right]$, then under the assumption of Theorem 3.3, we have

$$
\begin{aligned}
& \left(\epsilon_{\mu, \sigma, \jmath, \omega, x_{1}^{+}}^{\gamma, \delta, \kappa, \nu}\right)(z ; q)+\left(\epsilon_{\mu, \tau, \jmath, \omega, x_{2}^{-}}^{\gamma, \delta, \kappa, \nu}\right)(z ; q) \\
& \leq \frac{\|\hbar\|_{\infty}}{s+1}\left[\left(\zeta-x_{1}\right)\left(\epsilon_{\mu, \sigma-1, \jmath, x_{1}^{+}}^{\gamma, \delta, \kappa, \nu} 1\right)(z ; q)\left(\frac{1}{e^{\vartheta_{1} x_{1}}}+m \frac{1}{e^{\vartheta_{1} z}}\right)\right. \\
& \left.\quad+\left(x_{2}-z\right)\left(\epsilon_{\mu, \tau-1, \jmath, x_{2}^{-}}^{\gamma, \delta, \kappa, \nu}\right)(z ; q)\left(\frac{1}{e^{\vartheta_{2} x_{2}}}+m \frac{1}{e^{\vartheta_{2} z}}\right)\right] .
\end{aligned}
$$

Theorem 3.8. Suppose a real valued function $\hbar:\left[x_{1}, x_{1}+\eta\left(x_{2}, x_{1}\right)\right] \rightarrow \mathcal{R}$ is differentiable and $|\hbar|^{\prime}$ is exponentially $(s, m)$ preinvex, then the following fractional integral inequality 
for (2.9) and (2.10) holds:

$$
\begin{aligned}
& \epsilon_{\mu, \sigma+1, \jmath, \omega, x_{1}^{+}}^{\gamma, \delta, \kappa, \nu} \hbar(z ; q)+\epsilon_{\mu, \tau+1, \jmath, \omega,\left(x_{1}+\eta\left(x_{2}, x_{1}\right)\right)^{-}}^{\gamma, \delta, \kappa, \nu}(z ; q) \\
& \text { - } \epsilon_{\mu, \tau-1, j,\left(x_{1}+\eta\left(x_{2}, x_{1}\right)\right)^{\gamma}}^{\gamma, \delta, \kappa, \nu}(z ; q) \\
& \hbar\left(x_{1}+\eta\left(x_{2}, x_{1}\right)\right)-\epsilon_{\mu, \sigma-1, \jmath, x_{1}^{+}}^{\gamma, \delta, \kappa, \nu}(z ; q) \quad \hbar\left(x_{1}\right) \\
& \leq \frac{\left(\left(x_{1}+\eta\left(x_{2}, x_{1}\right)\right)-z\right) \epsilon_{\mu, \tau-1, j,\left(x_{1}+\eta\left(x_{2}, x_{1}\right)\right)^{-}}^{\gamma, \delta, \kappa, \nu}(z ; q)}{s+1} \\
& \frac{\left|\hbar^{\prime}\left(x_{1}+\eta\left(x_{2}, x_{1}\right)\right)\right|}{e^{\vartheta_{2}\left(x_{1}+\eta\left(x_{2}, x_{1}\right)\right)}}+m \frac{\left|\hbar^{\prime}(z)\right|}{e^{\vartheta_{2} z}} \\
& +\frac{\left(z-x_{1}\right) \epsilon_{\mu, \sigma-1, j, x_{1}^{+}}^{\gamma, \delta, \kappa, \nu}}{s+1} \frac{\left|\hbar^{\prime}\left(x_{1}\right)\right|}{e^{\vartheta_{1} x_{1}}}+m \frac{\left|\hbar^{\prime}(z)\right|}{e^{\vartheta_{1} z}},
\end{aligned}
$$

for all $z \in\left[x_{1} \cdot x_{1}+\eta\left(x_{2}, x_{1}\right)\right], \vartheta_{1}, \vartheta_{2} \in \mathcal{R}$.

Proof. Let $z \in\left[x_{1}, x_{1}+\eta\left(x_{2}, x_{1}\right)\right]$ and $\zeta \in\left[x_{1}, z\right)$, by using exponentially $(s, m)$ preinvexity of $|\hbar|^{\prime}$, we have

$$
\left|\hbar^{\prime}(\zeta)\right| \leq\left(\frac{z-\zeta}{z-x_{1}}\right)^{s} \frac{\left|\hbar^{\prime}\left(x_{1}\right)\right|}{e^{\vartheta_{1} x_{1}}}+m\left(\frac{\zeta-x_{1}}{z-x_{1}}\right)^{s} \frac{\left|\hbar^{\prime}(z)\right|}{e^{\vartheta_{1} z}} .
$$

Implies (3. 25 ), we have

$$
\hbar^{\prime}(\zeta) \leq\left(\frac{z-\zeta}{z-x_{1}}\right)^{s} \frac{\left|\hbar^{\prime}\left(x_{1}\right)\right|}{e^{\vartheta_{1} x_{1}}}+m\left(\frac{\zeta-x_{1}}{z-x_{1}}\right)^{s} \frac{\left|\hbar^{\prime}(z)\right|}{e^{\vartheta_{1} z}}
$$

Conducting product between (3.14) and (3.26), we get

$$
\begin{aligned}
& (z-\zeta)^{\sigma-1} E_{\mu, \sigma, j}^{\gamma, \delta, \kappa, \nu}\left(\omega(z-\zeta)^{\mu} ; q\right) \hbar^{\prime}(\zeta) \\
& \leq\left(z-x_{1}\right)^{\sigma-1} E_{\mu, \sigma, j}^{\gamma, \delta, \kappa, \nu}\left(\omega\left(z-x_{1}\right)^{\mu} ; q\right) \\
& \times \quad\left[\left(\frac{z-\zeta}{z-x_{1}}\right)^{s} \frac{\left|\hbar^{\prime}\left(x_{1}\right)\right|}{e^{\vartheta_{1} x_{1}}}+m\left(\frac{\zeta-x_{1}}{z-x_{1}}\right)^{s} \frac{\left|\hbar^{\prime}(z)\right|}{e^{\vartheta_{1} z}}\right] .
\end{aligned}
$$

Integrating the above inequality with respect to $\zeta$ from $x_{1}$ to $z$, we get

$$
\begin{aligned}
& \int_{x_{1}}^{z}(z-\zeta)^{\sigma-1} E_{\mu, \sigma, j}^{\gamma, \delta, \kappa, \nu}\left(\omega(z-\zeta)^{\mu} ; q\right) \hbar^{\prime}(\zeta) d \zeta \\
& \leq \int_{x_{1}}^{z}\left(z-x_{1}\right)^{\sigma-1} E_{\mu, \sigma, j}^{\gamma, \delta, \kappa, \nu}\left(\omega\left(z-x_{1}\right)^{\mu} ; q\right) \\
& \times \quad\left[\left(\frac{z-\zeta}{z-x_{1}}\right)^{s} \frac{\left|\hbar^{\prime}\left(x_{1}\right)\right|}{e^{\vartheta_{1} x_{1}}}+m\left(\frac{\zeta-x_{1}}{z-x_{1}}\right)^{s} \frac{\left|\hbar^{\prime}(z)\right|}{e^{\vartheta_{1} z}}\right] d \zeta \\
& =\frac{\left(z-x_{1}\right) E_{\mu, \sigma, j}^{\gamma, \delta, \nu}\left(\omega(z-\zeta)^{\mu} ; q\right)}{s+1}\left(\frac{\left|\hbar^{\prime}\left(x_{1}\right)\right|}{e^{\vartheta_{1} x_{1}}}+m \frac{\left|\hbar^{\prime}(z)\right|}{e^{\vartheta_{1} z}}\right) .
\end{aligned}
$$


Now solving left side of (3. 28 ) gives

$$
\begin{aligned}
& \int_{x_{1}}^{z}(z-\zeta)^{\sigma-1} E_{\mu, \sigma, j}^{\gamma, \delta, \kappa, \nu}\left(\omega(z-\zeta)^{\mu} ; q\right) \hbar^{\prime}(\zeta) d \zeta \\
& =\int_{0}^{z-x_{1}} \theta^{\sigma-1} E_{\mu, \sigma, j}^{\gamma, \delta, \kappa, \nu}\left(\omega \theta^{\mu} ; q\right) \hbar^{\prime}(z-\theta) d \theta \\
& =\left(z-x_{1}\right)^{\sigma-1} E_{\mu, \sigma, j}^{\gamma, \delta, \kappa, \nu}\left(\omega\left(z-x_{1}\right)^{\mu} ; q\right) \\
& \hbar\left(x_{1}\right)+\int_{0}^{z-x_{1}} \theta^{\sigma-2} E_{\mu, \sigma-1, j}^{\gamma, \delta, \kappa, \nu}\left(\omega \theta^{\mu} ; q\right) \hbar(z-\theta) d \theta .
\end{aligned}
$$

Again, substituting $z-\theta=\zeta$ in the second term and solving right hand side of the aforemenioned inequality, we have

$$
\begin{aligned}
& \int_{0}^{z-x_{1}} \theta^{\sigma-1} E_{\mu, \sigma-1, \jmath}^{\gamma, \delta, \kappa, \nu}\left(\omega \theta^{\mu} ; q\right) \hbar^{\prime}(z-\theta) d \theta \\
& =\left(z-x_{1}\right)^{\sigma-1} E_{\mu, \sigma, j}^{\gamma, \delta, \kappa, \nu}\left(\omega\left(z-x_{1}\right)^{\mu} ; q\right) \hbar\left(x_{1}\right) \\
& -\left(\epsilon_{\mu, \sigma-1, \jmath, \omega, x_{1}^{+}}^{\gamma, \delta, \kappa, \nu}\right)(z ; q) .
\end{aligned}
$$

Therefore (3. 28 ) gets the following form:

$$
\begin{aligned}
& \left(\left(\epsilon_{\mu, \sigma-1, \jmath, x_{1}^{+}}^{\gamma, \delta, \kappa, \nu} 1\right)(z ; q)\right) \hbar\left(x_{1}\right)-\left(\epsilon_{\mu, \sigma+1, \jmath, \omega, x_{1}^{+}}^{\gamma, \delta, \kappa, \nu}\right)(z ; q) \\
& \leq \frac{\left(z-x_{1}\right)\left(\epsilon_{\mu, \sigma-1, \jmath, x_{1}^{+}}^{\gamma, \delta, \kappa, \nu}\right)(z ; q)}{s+1}\left(\frac{\left|\hbar^{\prime}\left(x_{1}\right)\right|}{e^{\vartheta_{1} x_{1}}}+m \frac{\left|\hbar^{\prime}(z)\right|}{e^{\vartheta_{1} z}}\right) .
\end{aligned}
$$

Also from (3.25), we have

$$
\hbar^{\prime}(\zeta) \geq-\left(\left(\frac{z-\zeta}{z-x_{1}}\right)^{s} \frac{\left|\hbar^{\prime}\left(x_{1}\right)\right|}{e^{\vartheta_{1} x_{1}}}+m\left(\frac{\zeta-x_{1}}{z-x_{1}}\right)^{s} \frac{\left|\hbar^{\prime}(z)\right|}{e^{\vartheta_{1} z}}\right) .
$$

Adopting the same proceedure as we have done for (3. 26$)$, we obtain

$$
\begin{aligned}
& \left(\epsilon_{\mu, \sigma+1, \jmath, \omega, x_{1}^{+}}^{\gamma, \delta, \kappa, \nu}\right)(z ; q)-\left(\left(\epsilon_{\mu, \sigma-1, \jmath, x_{1}^{+}}^{\gamma, \delta, \kappa, \nu} 1\right)(z ; q)\right) \hbar\left(x_{1}\right) \\
& \leq \frac{\left(z-x_{1}\right)\left(\epsilon_{\mu, \sigma-1, \jmath, x_{1}^{+}}^{\gamma, \delta, \kappa, \nu} 1\right)(z ; q)}{s+1}\left(\frac{\left|\hbar^{\prime}\left(x_{1}\right)\right|}{e^{\vartheta_{1} x_{1}}}+m \frac{\left|\hbar^{\prime}(z)\right|}{e^{\vartheta_{1} z}}\right) .
\end{aligned}
$$

From (3. 29 ) and (3. 31 ), we get

$$
\begin{aligned}
& \left|\left(\epsilon_{\mu, \sigma+1, \jmath, \omega, x_{1}^{+}}^{\gamma, \delta, \kappa, \nu}\right)(z ; q)-\left(\left(\epsilon_{\mu, \sigma-1, \jmath, x_{1}^{+}}^{\gamma, \delta, \kappa, \nu} 1\right)(z ; q)\right) \hbar\left(x_{1}\right)\right| \\
& \leq \frac{\left(z-x_{1}\right)\left(\epsilon_{\mu, \sigma-1, \jmath, x_{1}^{+}}^{\gamma, \delta, \kappa, \nu} 1\right)(z ; q)}{s+1}\left(\frac{\left|\hbar^{\prime}\left(x_{1}\right)\right|}{e^{\vartheta_{1} x_{1}}}+m \frac{\left|\hbar^{\prime}(z)\right|}{e^{\vartheta_{1} z}}\right) .
\end{aligned}
$$


Now we let $x \in\left[x_{1}, x_{1}+\eta\left(x_{2}, x_{1}\right)\right]$ and $\zeta \in\left(z, x_{1}+\eta\left(x_{2}, x_{1}\right)\right]$. Then by exponentially $(s, m)$-preinvexity of $\left|\hbar^{\prime}\right|$, we have

$$
\begin{aligned}
& \left|\hbar^{\prime}(\zeta)\right| \leq \frac{\zeta-z}{x_{1}+\eta\left(x_{2}, x_{1}\right)-z} \frac{s}{\left|\hbar^{\prime}\left(x_{1}+\eta\left(x_{2}, x_{1}\right)\right)\right|} \\
& e^{\vartheta_{2} x_{2}} \\
& +m \frac{x_{1}+\eta\left(x_{2}, x_{1}\right)-\zeta}{x_{1}+\eta\left(x_{2}, x_{1}\right)-z} \frac{s \hbar^{\prime}(z) \mid}{e^{\vartheta_{2} z}}
\end{aligned}
$$

On the same lines as we have done for (3.14), (3.26) and (3.30), we can conclude (3. 17 ) and (3. 33 ), we have

$$
\begin{aligned}
& \epsilon_{\mu, \tau+1, \jmath, \omega,\left(x_{1}+\eta\left(x_{2}, x_{1}\right)\right)^{-}}^{\gamma, \delta, \kappa, \nu}(z ; q) \\
& -\quad \epsilon_{\mu, \sigma-1, j,\left(x_{1}+\eta\left(x_{2}, x_{1}\right)\right)^{-}}^{\gamma, \delta, \kappa, \nu}(z ; q) \quad \hbar\left(x_{1}+\eta\left(x_{2}, x_{1}\right)\right) \\
\leq & \frac{\left(\left(x_{1}+\eta\left(x_{2}, x_{1}\right)\right)-z\right) \epsilon_{\mu, \sigma-1, \jmath,\left(x_{1}+\eta\left(x_{2}, x_{1}\right)\right)^{-}}^{\gamma, \delta, \kappa, \nu}(z ; q)}{s+1} \\
& \times \frac{\left|\hbar^{\prime}\left(x_{1}+\eta\left(x_{2}, x_{1}\right)\right)\right|}{e^{\vartheta_{2}\left(x_{1}+\eta\left(x_{2}, x_{1}\right)\right)}+m \frac{\left|\hbar^{\prime}(z)\right|}{e^{\vartheta_{2} z}} .}
\end{aligned}
$$

From inequalities (3. 32 ) and (3. 34 ) via triangular inequality (3. 24 ) can be concluded.

The following corollaries are remarkable for Theorem 3.8.

Corollary 3.9. Choosing $\eta\left(x_{2}, x_{1}\right)=x_{2}-x_{1}$, then under the assumption of Theorem 3.8 , we have

$$
\begin{aligned}
& \mid\left(\epsilon_{\mu, \sigma+1, \jmath, \omega, x_{1}^{+}}^{\gamma, \delta, \kappa, \nu} \hbar\right)(z ; q)+\left(\epsilon_{\mu, \tau+1, \jmath, \omega, x_{2}^{-}}^{\gamma, \delta, \kappa, \nu}\right)(z ; q) \\
& \left.-\left(\epsilon_{\mu, \sigma-1, \jmath, x_{2}^{-}}^{\gamma, \delta, \kappa, \nu}\right)(z ; q) \hbar\left(x_{2}\right)-\left(\epsilon_{\mu, \sigma-1, \jmath, x_{1}^{+}}^{\gamma, \delta, \kappa, \nu} 1\right)(z ; q)\right) \hbar\left(x_{1}\right) \mid \\
& \leq \frac{\left(x_{2}-z\right)\left(\epsilon_{\mu, \sigma-1, \jmath, x_{2}^{-}}^{\gamma, \delta, \kappa, \nu} 1\right)(z ; q)}{s+1}\left(\frac{\left|\hbar^{\prime}\left(x_{2}\right)\right|}{e^{\vartheta_{2} x_{2}}}+m \frac{\left|\hbar^{\prime}(z)\right|}{e^{\vartheta_{2} z}}\right) \\
& \quad+\frac{\left(z-x_{1}\right)\left(\epsilon_{\mu, \sigma-1, \jmath, x_{1}^{+}}^{\gamma, \delta, \kappa, \nu}\right)(z ; q)}{s+1}\left(\frac{\left|\hbar^{\prime}\left(x_{1}\right)\right|}{e^{\vartheta_{1} x_{1}}}+m \frac{\left|\hbar^{\prime}(z)\right|}{e^{\vartheta_{1} z}}\right),
\end{aligned}
$$

for all $z \in\left[x_{1} . x_{2}\right], \vartheta_{1}, \vartheta_{2} \in \mathcal{R}$.

Corollary 3.10. Choosing $\eta\left(x_{2}, x_{1}\right)=x_{2}-x_{1}$, along with $s=m=1$ then under the assumption of Theorem 3.8, we have 


$$
\begin{aligned}
& \mid\left(\epsilon_{\mu, \sigma+1, \jmath, \omega, x_{1}^{+}}^{\gamma, \delta, \kappa, \nu}\right)(z ; q)+\left(\epsilon_{\mu, \tau+1, \jmath, \omega, x_{2}^{-}}^{\gamma, \delta, \kappa, \nu}\right)(z ; q) \\
& \left.-\left(\epsilon_{\mu, \sigma-1, \jmath, x_{2}^{-}}^{\gamma, \delta, \kappa, \nu}\right)(z ; q) \hbar\left(x_{2}\right)-\left(\epsilon_{\mu, \sigma-1, \jmath, x_{1}^{+}}^{\gamma, \delta, \kappa, \nu}\right)(z ; q)\right) \hbar\left(x_{1}\right) \mid \\
& \leq \frac{\left(x_{2}-z\right)\left(\epsilon_{\mu, \sigma-1, \jmath, x_{2}^{-}}^{\gamma, \delta, \kappa, \nu} 1\right)(z ; q)}{2}\left(\frac{\left|\hbar^{\prime}\left(x_{2}\right)\right|}{\left.e^{\vartheta_{2}\left(x_{1}+\eta\left(x_{2}, x_{1}\right)\right)}+\frac{\left|\hbar^{\prime}(z)\right|}{e^{\vartheta_{2} z}}\right)}\right. \\
& \quad+\frac{\left(z-x_{1}\right)\left(\epsilon_{\mu, \delta, \kappa, \nu}^{\gamma, \jmath, x_{1}^{+}} 1\right)(z ; q)}{2}\left(\frac{\left|\hbar^{\prime}\left(x_{1}\right)\right|}{e^{\vartheta_{1} x_{1}}}+\frac{\left|\hbar^{\prime}(z)\right|}{e^{\vartheta_{1} z}}\right),
\end{aligned}
$$

for all $z \in\left[x_{1}, x_{2}\right], \vartheta_{1}, \vartheta_{2} \in \mathcal{R}$.

Definition 3.11. Let $\hbar:\left[x_{1}, x_{1}+\eta\left(x_{2}, x_{1}\right)\right] \rightarrow \mathcal{R}$ be a function, we say $\hbar$ is exponentially symmetric about $\frac{2 x_{1}+\eta\left(x_{2}, x_{1}\right)}{2}$, if

$$
\frac{\hbar(z)}{e^{\vartheta_{1} z}}=\frac{\hbar\left(2 x_{1}+\eta\left(x_{2}, x_{1}\right)-z\right)}{e^{\vartheta_{1}\left(\left(2 x_{1}+\eta\left(x_{2}, x_{1}\right)-z\right)\right)}}, \vartheta_{1} \in \mathcal{R}
$$

Now we demonstrate the following lemma which will be helpful to produce our coming result.

Lemma 3.12. Let $\hbar:\left[x_{1}, x_{1}+\eta\left(x_{2}, x_{1}\right)\right] \rightarrow \mathcal{R}$ be an exponentially symmetric, then

$$
\hbar\left(\frac{2 x_{1}+\eta\left(x_{2}, x_{1}\right)}{2}\right) \leq \frac{(1+m) \hbar(z)}{2^{s} e^{\vartheta_{1} z}}, \vartheta_{1} \in \mathcal{R}
$$

Proof. Since $\hbar$ is exponentially $(s, m)$-preinvex, therefore

$$
\begin{aligned}
& \hbar\left(\frac{2 x_{1}+\eta\left(x_{2}, x_{1}\right)}{2}\right) \leq \frac{\hbar\left(x_{1}+\zeta \eta\left(x_{2}, x_{1}\right)\right)}{2^{s} e^{\vartheta_{1} \hbar\left(x_{1}+\zeta \eta\left(x_{2}, x_{1}\right)\right)}} \\
& +m \frac{\hbar\left(x_{1}+(1-\zeta) \eta\left(x_{2}, x_{1}\right)\right)}{2^{s} e^{\vartheta_{1} \hbar\left(x_{1}+(1-\zeta) \eta\left(x_{2}, x_{1}\right)\right)}} .
\end{aligned}
$$

Let $z=x_{1}+\zeta \eta\left(x_{2}, x_{1}\right)$, where $z \in\left[x_{1}, x_{1}+\eta\left(x_{2}, x_{1}\right)\right]$. Then we have $2 x_{1}+\eta\left(x_{2}, x_{1}\right)=$ $x_{1}+(1-\zeta) \eta\left(x_{2}, x_{1}\right)$ and we have

$$
\hbar\left(\frac{2 x_{1}+\eta\left(x_{2}, x_{1}\right)}{2}\right) \leq \frac{\hbar(z)}{2^{s} e^{\vartheta_{1} z}}+m \frac{\hbar\left(2 x_{1}+\eta\left(x_{2}, x_{1}\right)-z\right)}{2^{s} e^{\vartheta_{1}\left(2 x_{1}+\eta\left(x_{2}, x_{1}\right)-z\right)}} .
$$

Now using that $\hbar$ is exponentially symmetric we will get (3. 36 ).

Theorem 3.13. Suppose a real valued function $\hbar:\left[x_{1}, x_{1}+\eta\left(x_{2}, x_{1}\right)\right] \rightarrow \mathcal{R}$ is positive, exponentially $(s, m)$-preinvex and expoennetially symmetric about $\frac{2 x_{1}+\eta\left(x_{2}, x_{1}\right)}{2}$, then the 
following fractional integral inequality for (2.9) and (2.10) holds:

$$
\begin{aligned}
& \frac{2^{s}}{1+m} \hbar\left(\frac{2 x_{1}+\eta\left(x_{2}, x_{1}\right)}{2}\right) \\
\times & {\left[\left(e^{\vartheta_{1} x_{1}}\right)\left(\epsilon_{\mu, \tau+1, \jmath, \omega,\left(x_{1}+\eta\left(x_{2}, x_{1}\right)\right)^{-}}^{\gamma, \delta, \kappa, \nu}\right)\left(x_{1} ; q\right)\right.} \\
& \left.+e^{\vartheta_{2}\left(x_{1}+\eta\left(x_{2}, x_{1}\right)\right.}\left(\epsilon_{\mu, \tau+1, \jmath, \omega, x_{1}^{+}}^{\gamma, \delta, \kappa, \nu}\right)\left(x_{1}+\eta\left(x_{2}, x_{1}\right) ; q\right)\right] \\
\leq & \left(\epsilon_{\mu, \tau+1, \jmath, \omega, x_{1}^{+}}^{\gamma, \delta, \kappa, \nu}\right)\left(x_{1}+\eta\left(x_{2}, x_{1}\right) ; q\right) \\
+ & \left(\epsilon_{\mu, \tau+1, \jmath, \omega,\left(x_{1}+\eta\left(x_{2}, x_{1}\right)\right)^{-}}^{\gamma, \delta, \kappa, \nu}\right)\left(x_{1} ; q\right) \\
\leq & \frac{\eta\left(x_{2}, x_{1}\right)}{s+1}\left(\frac{\hbar\left(x_{1}+\eta\left(x_{2}, x_{1}\right)\right)}{e^{\vartheta_{2}\left(x_{1}+\eta\left(x_{2}, x_{1}\right)\right)}}\right. \\
+ & m \frac{\hbar\left(x_{1}\right)}{\left.e^{\vartheta_{1} x_{1}}\right)\left[\left(\epsilon_{\mu, \sigma-1, \jmath, \omega, x_{1}^{-}}^{\gamma, \delta, \kappa, \nu}\right)\left(x_{1}+\eta\left(x_{2}, x_{1}\right) ; q\right)\right.} \\
& \left.+\left(\epsilon_{\mu, \tau-1, \jmath, \omega,\left(x_{1}+\eta\left(x_{2}, x_{1}\right)\right)^{-}}^{\gamma, \delta, \kappa, \nu}\right)\left(x_{1} ; q\right)\right] .
\end{aligned}
$$

Proof. For $z \in\left[x_{1}, x_{1}+\eta\left(x_{2}, x_{1}\right)\right]$, we have

$$
\begin{aligned}
& \left(z-x_{1}\right)^{\tau} E_{\mu, \tau+1, j}^{\gamma, \delta, \kappa, \nu}\left(\omega\left(z-x_{1}\right)^{\mu} ; q\right) \\
& \leq\left(\eta\left(x_{2}, x_{1}\right)\right)^{\tau} E_{\mu, \tau+1, j}^{\gamma, \delta, \kappa, \nu}\left(\omega\left(\eta\left(x_{2}, x_{1}\right)^{\mu} ; q\right), \tau>0\right.
\end{aligned}
$$

As $\hbar$ is exponentially $(s, m)$-preinvex so for $z \in\left[x_{1}, x_{1}+\eta\left(x_{2}, x_{1}\right)\right]$, we have

$$
\begin{aligned}
& \hbar(z) \leq\left(\frac{z-x_{1}}{\eta\left(x_{2}, x_{1}\right)}\right)^{s} \frac{\hbar\left(x_{1}+\eta\left(x_{2}, x_{1}\right)\right)}{e^{\vartheta}\left(x_{1}+\eta\left(x_{2}, x_{1}\right)\right)} \\
& +m\left(\frac{\left(x_{1}+\eta\left(x_{2}, x_{1}\right)\right)-z}{\eta\left(x_{2}, x_{1}\right)}\right)^{s} \frac{\hbar\left(x_{1}\right)}{e^{\vartheta_{1} x_{1}}}
\end{aligned}
$$

Conducting product between (3.40) and (3.41) and then integrating with respect to the variable $z$ from $x_{1}$ and $x_{2}$, we get

$$
\begin{array}{r}
\int_{x_{1}}^{x_{2}}\left(z-x_{1}\right)^{\tau} E_{\mu, \tau+1, \jmath}^{\gamma, \delta, \kappa, \nu}\left(\omega\left(z-x_{1}\right)^{\mu} ; q\right) \hbar(z) d z \\
\leq\left(\eta\left(x_{2}, x_{1}\right)\right)^{\tau} E_{\mu, \tau+1, \jmath}^{\gamma, \delta, \kappa, \nu}\left(\omega\left(\eta\left(x_{2}, x_{1}\right)^{\mu} ; q\right)\right. \\
\quad \times \int_{x_{1}}^{x_{2}}\left[\left(\frac{z-x_{1}}{\eta\left(x_{2}, x_{1}\right)}\right) \frac{\hbar\left(x_{1}+\eta\left(x_{2}, x_{1}\right)\right)}{e^{\vartheta_{2}\left(x_{1}+\eta\left(x_{2}, x_{1}\right)\right)}}\right. \\
\left.\quad+m\left(\frac{\left(x_{1}+\eta\left(x_{2}, x_{1}\right)\right)-z}{\eta\left(x_{2}, x_{1}\right)}\right)^{s} \frac{\hbar\left(x_{1}\right)}{e^{\vartheta_{1} x_{1}}}\right] d z .
\end{array}
$$


from which we have

$$
\begin{aligned}
& \left(\epsilon_{\mu, \tau, j, \omega,\left(x_{1}+\eta\left(x_{2}, x_{1}\right)\right)^{-}}^{\gamma, \delta, \kappa, \nu}\right)\left(x_{1}, q\right) \\
& \leq \frac{\left(\eta\left(x_{2}, x_{1}\right)\right)^{\tau} E_{\mu, \tau, j}^{\gamma, \delta, \kappa, \nu}\left(\omega\left(\eta\left(x_{2}, x_{1}\right)\right)^{\mu} ; q\right)}{s+1} \\
& \times\left(\frac{\hbar\left(x_{1}+\eta\left(x_{2}, x_{1}\right)\right)}{e^{\vartheta_{2}\left(x_{1}+\eta\left(x_{2}, x_{1}\right)\right)}}+m \frac{\hbar\left(x_{1}\right)}{e^{\vartheta_{1} x_{1}}}\right), \\
& =\frac{\eta\left(x_{2}, x_{1}\right)}{s+1}\left(\epsilon_{\mu, \tau-1, j, \omega,\left(x_{1}+\eta\left(x_{2}, x_{1}\right)\right)^{-}}^{\gamma, \delta, \kappa, \nu}\right)\left(x_{1} ; q\right) \\
& \times\left(\frac{\hbar\left(x_{1}+\eta\left(x_{2}, x_{1}\right)\right)}{e^{\vartheta_{2}\left(x_{1}+\eta\left(x_{2}, x_{1}\right)\right)}}+m \frac{\hbar\left(x_{1}\right)}{e^{\vartheta_{1} x_{1}}}\right) .
\end{aligned}
$$

Analogously for $z \in\left[x_{1}, x_{1}+\eta\left(x_{2}, x_{1}\right)\right]$, we have

$$
\begin{aligned}
& \left(x_{1}+\eta\left(x_{2}, x_{1}\right)-z\right)^{\sigma} E_{\mu, \sigma+1, \jmath}^{\gamma, \delta, \kappa, \nu}\left(\omega\left(x_{1}+\eta\left(x_{2}, x_{1}\right)\right) ; q\right) \\
& \leq\left(\eta\left(x_{2}, x_{1}\right)\right)^{\sigma} E_{\mu, \sigma+1, \jmath}^{\gamma, \delta, \kappa, \nu}\left(\omega\left(\eta\left(x_{2}, x_{1}\right)\right)^{\mu} ; q\right), \quad \vartheta_{1}>0 .
\end{aligned}
$$

Conducting product (3. 40 ) and (3. 43 ) and then integrating with respect to $z$ with respect to $x_{1}$ and $x_{2}$, we get

$$
\begin{aligned}
& \int_{x_{1}}^{x_{2}}\left(x_{1}+\eta\left(x_{2}, x_{1}\right)-z\right)^{\sigma} E_{\mu, \sigma+1, j}^{\gamma, \delta, \kappa, \nu}\left(\omega\left(x_{1}+\eta\left(x_{2}, x_{1}\right)\right)^{\mu} ; q\right) \hbar(z) d z \\
& \leq\left(\eta\left(x_{2}, x_{1}\right)\right)^{\sigma} E_{\mu, \sigma+1, j}^{\gamma, \delta, \kappa, \nu}\left(\omega\left(\eta\left(x_{2}, x_{1}\right)\right)^{\mu} ; q\right) \\
& \times \quad \int_{x_{1}}^{x_{2}}\left[\left(\frac{z-x_{1}}{\eta\left(x_{2}, x_{1}\right)}\right)^{s} \frac{\hbar\left(x_{1}+\eta\left(x_{2}, x_{1}\right)\right)}{e^{\vartheta_{2}\left(x_{1}+\eta\left(x_{2}, x_{1}\right)\right)}}\right. \\
& \left.+m\left(\frac{\left(x_{1}+\eta\left(x_{2}, x_{1}\right)\right)-z}{\eta\left(x_{2}, x_{1}\right)}\right)^{s} \frac{\hbar\left(x_{1}\right)}{e^{\vartheta_{1} x_{1}}}\right] d z .
\end{aligned}
$$

From which we have

$$
\begin{aligned}
& \epsilon_{\mu, \sigma, \jmath \omega, x_{1}^{+}}^{\gamma, \delta, \kappa, \nu}\left(x_{1}+\eta\left(x_{2}, x_{1}\right): q\right) \\
\leq & \frac{\left(\eta\left(x_{2}, x_{1}\right)\right)^{\sigma} E_{\mu, \sigma, j}^{\gamma, \delta, \kappa}\left(\omega\left(\eta\left(x_{2}, x_{1}\right)\right)^{\mu} ; q\right)}{s+1} \\
\times & \frac{\hbar\left(x_{1}+\eta\left(x_{2}, x_{1}\right)\right)}{e^{\vartheta_{2}\left(x_{1}+\eta\left(x_{2}, x_{1}\right)\right)}+m \frac{\hbar\left(x_{1}\right)}{e^{\vartheta} x_{1}}} \\
= & \frac{\eta\left(x_{2}, x_{1}\right)}{s+1} \epsilon_{\mu, \sigma-1, \jmath, \omega, x_{1}^{-}}^{\gamma, \delta, \kappa, \nu}\left(x_{1}+\eta\left(x_{2}, x_{1}\right) ; q\right) \\
\times & \frac{\hbar\left(x_{1}+\eta\left(x_{2}, x_{1}\right)\right)}{e^{\vartheta_{2}\left(x_{1}+\eta\left(x_{2}, x_{1}\right)\right)}}+m \frac{\hbar\left(x_{1}\right)}{e^{\vartheta_{1} x_{1}}} .
\end{aligned}
$$


Summing up (3. 42 ) and (3. 45$)$, we attain

$$
\begin{aligned}
& \left(\epsilon_{\mu, \tau, \jmath, \omega,\left(x_{1}+\eta\left(x_{2}, x_{1}\right)\right)^{-}}^{\gamma, \delta, \kappa, \nu}\right)\left(x_{1}, q\right) \\
& +\left(\epsilon_{\mu, \sigma, \jmath \omega, x_{1}^{+}}^{\gamma, \delta, \kappa}\right)\left(x_{1}+\eta\left(x_{2}, x_{1}\right): q\right) \\
& \leq \frac{\eta\left(x_{2}, x_{1}\right)}{s+1}\left(\frac{\hbar\left(x_{1}+\eta\left(x_{2}, x_{1}\right)\right)}{e^{\vartheta_{2}\left(x_{1}+\eta\left(x_{2}, x_{1}\right)\right)}}\right. \\
& \left.+m \frac{\hbar\left(x_{1}\right)}{e^{\vartheta_{1} x_{1}}}\right)\left[\left(\epsilon_{\mu, \sigma-1, \jmath, \omega, x_{1}^{-}}^{\gamma, \delta, \kappa, \nu}\right)\left(x_{1}+\eta\left(x_{2}, x_{1}\right) ; q\right)\right. \\
& \left.\quad+\left(\epsilon_{\mu, \tau-1, \jmath, \omega,\left(x_{1}+\eta\left(x_{2}, x_{1}\right)\right)^{-}}^{\gamma, \delta, \kappa, \nu}\right)\left(x_{1} ; q\right)\right] .
\end{aligned}
$$

Taking product (3. 36$)$ with $\left(z-x_{1}\right)^{\tau} E_{\mu, \tau+1, \jmath}^{\gamma, \delta, \kappa, \nu}\left(\omega\left(z-x_{1}\right)^{\mu} ; q\right)$ and integrating with respect to the variable $z$ from $x_{1}$ and $x_{2}$, we have

$$
\begin{aligned}
& \hbar\left(\frac{2 x_{1}+\eta\left(x_{2}, x_{1}\right)}{2}\right) \int_{x_{1}}^{x_{2}}\left(z-x_{1}\right)^{\tau} E_{\mu, \tau+1, j}^{\gamma, \delta, \kappa, \nu}\left(\omega\left(z-x_{1}\right)^{\mu} ; q\right) d z \\
& \leq \frac{(1+m)}{2^{s}} \int_{x_{1}}^{x_{2}}\left(z-x_{1}\right)^{\tau} E_{\mu, \tau+1, j}^{\gamma, \delta, \kappa, \nu}\left(\omega\left(z-x_{1}\right)^{\mu} ; q\right) \frac{\hbar(z)}{e^{\vartheta_{1} z}} d z .
\end{aligned}
$$

Using Definition 2.8, we get

$$
\begin{aligned}
& \hbar\left(\frac{2 x_{1}+\eta\left(x_{2}, x_{1}\right)}{2}\right)\left(\epsilon_{\mu, \tau+1, \jmath, \omega,\left(x_{1}+\eta\left(x_{2}, x_{1}\right)\right)^{-}}^{\gamma, \delta, \kappa, \nu}\right)\left(x_{1} ; q\right) \\
& \leq \frac{(1+m)}{2^{s} e^{\vartheta_{1} x_{1}}}\left(\epsilon_{\mu, \tau+1, \jmath, \omega,\left(x_{1}+\eta\left(x_{2}, x_{1}\right)\right)^{-}}^{\gamma, \delta, \kappa, \nu}\right)\left(x_{1} ; q\right)
\end{aligned}
$$

Taking product (3. 36$)$ with $\left(x_{1}+\eta\left(x_{2}, x_{1}\right)-z\right)^{\sigma} E_{\mu, \sigma, j}^{\gamma, \delta, \kappa, \nu}\left(\omega\left(x_{1}+\eta\left(x_{2}, x_{1}\right)\right)^{\mu} ; q\right)$ and integrating with respect to the variable $z$ from $x_{1}$ and $x_{2}$, we have

$$
\begin{aligned}
& \hbar\left(\frac{2 x_{1}+\eta\left(x_{2}, x_{1}\right)}{2}\right)\left(\epsilon_{\mu, \tau+1, \jmath, \omega, x_{1}^{+}}^{\gamma, \delta, \kappa, \nu}\right)\left(x_{1}+\eta\left(x_{2}, x_{1}\right) ; q\right) \\
& \leq \frac{(1+m)}{2^{s} e^{\vartheta_{2}\left(x_{1}+\eta\left(x_{2}, x_{1}\right)\right.}}\left(\epsilon_{\mu, \tau+1, \jmath, \omega, x_{1}^{+}}^{\gamma, \delta, \kappa, \nu}\right)\left(x_{1}+\eta\left(x_{2}, x_{1}\right) ; q\right) .
\end{aligned}
$$

Summing up (3. 47 ) and (3. 48$)$, we get

$$
\begin{aligned}
& \frac{2^{s}}{1+m} \hbar \frac{2 x_{1}+\eta\left(x_{2}, x_{1}\right)}{2} \\
& \times\left[\left(e^{\vartheta_{1} x_{1}}\right) \epsilon_{\mu, \tau+1, \jmath, \omega,\left(x_{1}+\eta\left(x_{2}, x_{1}\right)\right)^{-}}^{\gamma, \delta, \kappa, \nu}\left(x_{1} ; q\right)\right. \\
& \left.\quad+e^{\vartheta_{2}\left(x_{1}+\eta\left(x_{2}, x_{1}\right)\right.} \epsilon_{\mu, \tau+1, \jmath, \omega, x_{1}^{+}}^{\gamma, \delta, \kappa, \nu}\left(x_{1}+\eta\left(x_{2}, x_{1}\right) ; q\right)\right] \\
& \leq \epsilon_{\mu, \tau+1, \jmath, \omega, x_{1}^{+}}^{\gamma, \delta, \kappa, \nu}\left(x_{1}+\eta\left(x_{2}, x_{1}\right) ; q\right) \\
& +\epsilon_{\mu, \tau+1, \jmath, \omega,\left(x_{1}+\eta\left(x_{2}, x_{1}\right)\right)^{-}}^{\gamma, \delta, \kappa}\left(x_{1} ; q\right)
\end{aligned}
$$

By combining (3. 46 ) and (3. 50 ), we get inequality (3. 39). 
Corollary 3.14. Choosing $\eta\left(x_{2}, x_{1}\right)=x_{2}-x_{1}$, then under the assumption of Theorem 3.13, we have

$$
\begin{aligned}
& \frac{2^{s}}{1+m} \hbar\left(\frac{\left.x_{1}+x_{2}\right)}{2}\right)\left[e^{\vartheta_{1} x_{1}}\left(\epsilon_{\mu, \tau+1, \jmath, \omega, x_{2}^{-}}^{\gamma, \delta, \kappa, \nu}\right)\left(x_{1} ; q\right)\right. \\
& \left.\left.+e^{\vartheta_{2} x_{2}}\left(\epsilon_{\mu, \tau+1, \jmath, \omega, x_{1}^{+}}^{\gamma, \delta, \kappa, \nu}\right)\left(x_{2}\right) ; q\right)\right] \\
\leq & \left.\left(\epsilon_{\mu, \tau+1, \jmath, \omega, x_{1}^{+}}^{\gamma, \delta, \kappa, \nu}\right)\left(x_{2}\right) ; q\right)+\left(\epsilon_{\mu, \tau+1, \jmath, \omega, x_{2}^{-}}^{\gamma, \delta, \kappa, \nu}\right)\left(x_{1} ; q\right) \\
\leq & \frac{\left(x_{2}-x_{1}\right)}{s+1}\left(\frac{\hbar\left(x_{2}\right)}{e^{\vartheta_{2} x_{2}}}+m \frac{\hbar\left(x_{1}\right)}{e^{\vartheta_{1} x_{1}}}\right) \\
\times & {\left[\left(\epsilon_{\mu, \sigma-1, \jmath, \omega, x_{1}^{+}}^{\gamma, \delta, \kappa, \nu}\right)\left(x_{2} ; q\right)+\left(\epsilon_{\mu, \tau-1, \jmath, \omega, x_{2}^{-}}^{\gamma, \delta, \kappa, \nu}\right)\left(x_{1} ; q\right)\right] }
\end{aligned}
$$

\section{CONCLUSION}

In this study, we have introduced the more general form of fractional integral inequalities by choosing the appropriate and suitable choice of parameters can be attained. For instance, choosing $q=0$, and $\jmath=\delta=1$ in Definition 2.8 our results reduces to the results for fractional integral operators defined by Salim and Faraj [33] and Rahman et al. [6], respectively. Moreover, for $q=0$ and $\jmath=\delta=1$, our resutlts reduces to fractional integral operators defined by Shukla and Prajapati in [47], choosing $q=0$ and $\jmath=\delta=\kappa=1$, for fractional integral operators defined by Prabhakar in [5], under some certain conditions defined by exponentially $(s, m)$-preinvex functions. By choosing $q=\omega=0$ fractional integral inequalities for Riemann-Liouville fractional integrals. All the results hold for $s$ preinvex, $m$-preinvex, exponentially preinvex, exponentially $s$-preinvex as well as convex functions. The results can be used to study some new and know results having application in scientific and technological fields.

\section{ACKNOWLEDGMENTS}

I would like to thank my supervisor, Prof. Muhammad Noor, for his guidance, encouragement and advice. I have also been extremely thankful to my dear friend Dr. Saima Rashid for her complete support and help during my work.

\section{Authors COnTributions}

Both the authors worked jointly. They all read and approved the final manuscript.

\section{REFERENCES}

[1] T. Abdeljawad, On conformable fractional calculus, J. Comput. Appl. Math. 279 (2015) : 57-66.

[2] T. Abdeljawad and D. Baleanu, Monotonicity results for fractional difference operators with discrete exponential kernels, Adv. Differ. Equ.78 2017. doi.org/10.1186/s13662-017-1126-1.

[3] F. Jarad, T. Abdeljawad and J. Alzabut, Generalized fractional derivatives generated by a class of local proportional derivatives, Eur. Phys. J. Spec. Top. 226 (2017) 3457-3471.

[4] F. Jarad, E. Ugurlu, T. Abdeljawad and D. Baleanu, On a new class of fractional operators, Adv. Differ. Equ.47 (2017).

[5] T. R. Prabhakar, A singular integral equation with a generalized Mittag-Leffler function in the kernel, Yokohama Math. J. 19 (1971) 7-15. 
[6] G. Rahman, D.Baleanu, M.A. Qurashi, S. D. Purohit ,Mubeen and M. Arshad, The extended Mittag-Leffler function via fractional calculus, J. Nonlinear Sci. Appl. 10 (2017) 4244-4253.

[7] S.Rashid, M.A. Noor and K.I. Noor,Inequalities pertaining fractional approach through exponentially convex functions, Fractal Fract. 3, No. 37. (2019) doi:10.3390/fractalfract3030037.

[8] M. K. Wang, M.Y. Hong, Y. F. Xu, Z. H. Shen and Y. M. Chu, Inequalities for generalized trigonometric and hyperbolic functions with one parameter, J. Math. Inequal.14, No. 1 (2020) 1-21. doi.org/10.7153/jmi2020-14-01.

[9] W. M. Qian, W. Zhang and Y. M. Chu, Bounding the convex combination of arithmetic and integral means in terms of one-parameter harmonic and geometric means, Miskolc Math. Notes. 20, No. 2 (2019) 1157-1166. doi.org/10.18514/MMN.2019.2334.

[10] S. Khan , M.A. Khan and Y.M. Chu, Converses of the Jensen inequality derived from the Green functions with applications in information theory, Math. Methods Appl. Sci. 43, No.5 (2020) 2577-2587. doi.org/10.1002/mma.6066.

[11] T. H. Zhao, L. Shi, Y.M. Chu, Convexity and concavity of the modified Bessel functions of the first kind with respect to Hölder means, RACSAM. 114, No.2 (2020) doi.org/10.1007/s13398-020-00825-3.

[12] A. Iqbal, M. A. Khan, S. Ullah and Y.M. Chu, Some New Hermite-Hadamard-type inequalities associated with conformable fractional integrals and their applications,J. Funct. Spaces. 2020 (2020) 9845407

[13] M. K. Wang, Z. Y. He and Y. M. Chu, Sharp power mean inequalities for the generalized elliptic integral of the first kind, Comput. Methods Funct. Theory, 20, No. 1(2020) 111-124.

[14] X. M. Hu , J.F. Tian, Y.M. Chu and X. Y. Lu, On Cauchy-Schwarz inequality for N-tuple diamond-alpha integral, J. Inequal. Appl. 2020: 8 (2020) doi.org/10.1186/s13660-020-2283-4.

[15] Z. H. Yang, W. M. Qian, W. Zhang and Y.M. Chu, Notes on the complete elliptic integral of the first kind, Math. Inequal. Appl. 23, No.1 (2020) 77-93.

[16] S. S. Dragomir, Refinements of the Hermite-Hadamard integral inequality for log-convex functions, The Australian. Math. Society Gazette, 28, No.3 (2001) 129-134.

[17] S.S. Dragomir and S. Fitzpatrick, The Hadamard's inequality for s-convex functions in the second sense, Demonstr. Math. 32, No 4 (1999)687-696.

[18] G. Farid, K. N. Khan, N. Latif, A. U. Rehman, S. Mehmood, General fractional integral inequalities for convex and $m$-convex functions via an extended generalized Mittag-Leffler function, J. Inequal. Appl.2018 (2018) 243.

[19] S. Rashid M.A. Noor, K.I. Noor and Y. M. Chu, Ostrowski type inequalities in the sense of generalized K-fractional integral operator for exponentially convex functions, AIMS Math. 5, No.3 (2020) 26292645. doi.org/10.3934/math.2020171.

[20] M. A. Latif, S. Rashid, S. S. Dragomir and Y. M. Chu, HermiteHadamard type inequalities for coordinated convex and qausi-convex functions and their applications, J. Inequal. Appl. (2019): Article ID 317 doi.org/10.1186/s13660-019-2272-7.

[21] S. Rashid, Z. Hammouch, K. Kalsoom, R. Ashraf and Y. M. Chu, New investigation on the generalized K-fractional integral operators, Front. Phys. 8:25 (2020) doi:10.3389/fphy.2020.00025.

[22] S. Rashid, R. Ashraf, M.A. Noor, K. I. Noor and Y. M. Chu, New weighted generalizations for differentiable exponentially convex mapping with application, AIMS Math.5, No.4 (2020) 3525-3546. doi:10.3934/math.2020229.

[23] S. Rashid, M.A. Noor, K.I. Noor,F. Safdar and Y. M. Chu, Hermite-Hadamard type inequalities for the class of convex functions on time scale, Mathematics, 7 (2019) 956 doi:10.3390/math7100956.

[24] H. Kalsoom, S. Rashid, M. Idrees, Y. M. Chu and D. Baleanu, Two-variable quantum integral inequalities of Simpson-type based on higher-order generalized strongly preinvex and quasi-preinvex functions, Symmetry, 12 (2020) 51. doi:10.3390/sym12010051.

[25] S. Rashid, H. Kalsoom, Z. Hammouch, R. Ashraf, D. Baleanu and M. Y. Chu, New multi-parametrized estimates having pth-order differentiability in fractional calculus for predominating $h$-convex functions in Hilbert space, Symmetry, 12 (2020) 222. doi:10.3390/sym12020222.

[26] S. Rashid, H. Kalsoom, M. Idrees, F. Safdar, S.Akram, D. Baleanu and Y. M. Chu, Post quantum integral inequalities of Hermite-Hadamard-type associated with Co-ordinated higher-order generalized strongly preInvex and quasi-pre-invex mappings Symmetry, 12 (2020) 443 doi:10.3390/sym12030443.

[27] S. Rashid, F. Jarad, M. A. Noor, K. I. Noor, D. Baleanu and B. J. Liu, On Gruss inequalities within generalized K-fractional integrals, Adv. Differ. Eq. 2020 (2020)203. doi.org/10.1186/s13662-020-02644-7. 
[28] S. Rashid, T. Abdeljawad, F. Jarad and M. A. Noor, Some estimates for generalized Riemann-Liouville fractional integrals of exponentially convex functions and their applications, Mathematics, 7, No.9 (2019) 807. doi.org/10.3390/math7090807.

[29] M. U. Awan, M. A. Noor and K. I. Noor, Hermite-Hadamard inequalities for exponentially convex functions, Appl. Math. Inf. Sci. 12, No. 2 (2018) 405-409.

[30] S. N . Bernstein, Sur les fonctions absolument monotones, Acta. Math. 52 (1929) 1-66.

[31] M. Avriel, Solution of certain nonlinear programs involving r-convex functions, J. Optim, Theory. Appl. 11, No. 2 (1973) 159-174.

[32] G. Alirezaei and R. Mathar, On exponentially concave functions and their impact in information theory, J. Inf. Theory. Appl. 9 (2018) 265-274.

[33] T. O. Salim, A.W. Faraj, Generalization of Mittag-Leffler function and integral operator associated with fractional calculus, J. Frac. Calc. Appl. 3, NO. 5 (2012) 1-13.

[34] E. Set, M.E. Ozdemir and S. S. Dragomir, On the Hermite-Hadamard inequality and other integral inequalities involving two functions, J. Inequal. Appl. 2010(2010) Article ID 148102.

[35] B.D. Craven, Duality for generalized convex fractional programs. In Generalized Convexity in Optimization and Economics, Schaible, S., Ziemba, T., Eds.; Academic Press: San Diego, CA, USA.(1981) 473-489.

[36] M.A. Hanson, On sufficiency of the Kuhn-Tucker conditions, J. Math. Anal. Appl. 80 (1981) 545-550.

[37] T. Weir and B. Mond, Preinvex functions in multi objective optimization, J. Math. Anal. Appl. 136 (1988) 29-38.

[38] M.A. Noor, Hermite-Hadamard integral inequalities for log-preinvex functions, J. Math. Anal. Approx. Theory, 2 (2007) 126-131.

[39] S, R. Mohan, and S.K. Neogy, On invex sets and preinvex functions, J. Math. Anal. Appl. 189 (1995) 901908.

[40] S. Mititelu, Invex sets, Stud. Cercet. Stiint. Univ. Bacau Ser. Mat. 46 (1994) 529-532.

[41] G. Toader, Some generalizations of the convexity, Proc. Colloq. Approx. Optim. (1984) 329-338.

[42] G.M. Leffler, Sur la nouvelle fonction $E_{\alpha}(x)$, Comptes Rendus Hebdomadaires des Seances de 1 Academie des Sciences Paris, 137 (1903)554-558.

[43] M. Andric, G. Farid and J. Pecaric, A further extension of Mittag-Leffler function, Fract. Calc. Appl. Anal. 21, No.5 (2018) 1377-1395.

[44] M. Arshad, J. Choi, S. Mubeen, K. S. Nisar and G. Rahman , A new extension of Mittag-Leffler function, Commun. Korean Math. Soc. 33, No. 2 (2018) 549-560.

[45] H. J. Haubold and A. M. Mathai, Saxena R K. Mittag-Leffler functions and their applications, J. Appl. Math. 2011(2011) Article ID 298628.

[46] H. Hudzik and L. Maligranda, Some remarks on s-convex functions, Aequ. Math. 48 (1994) 100-111.

[47] A. K. Shukla and J. C. Prajapati, On a generalization of Mittag-Leffler function and its properties, J. Math. Anal. Appl.336 (2007) 797-811.

[48] H. M. Srivastava and Z. Tomovski, Fractional calculus with an integral operator containing generalized Mittag-Leffler function in the kernel, Appl. Math. Comput.211, No. 1 (2009) 198-210.

[49] A. M. Alghamdi, S. Gala and M.A. Ragusa, A regularity criterion for local strong solutions to the $3 D$ StokesMHD equations, Ann. Pol. Math.,124, No. 3 (2020) 247-255.

[50] E. Set, A. O. Akdemir and E. A. Alan, Hermite-Hadamard and HermiteHadamard Fejer type inequalities involving fractional integral operators, Filomat, 33, No. 8 (2019) 2367-2380.

[51] S. I. Butt, A. O. Akdemir, M. Nadeem, N. Mlaiki, I. Iscan and T. Abdeljawad, (n,m)-Harmonically polynomial convex functions and some Hadamard type inequalities on the co-ordinates, AIMS Math.,6, No.5 (2021) 4677-4690.

[52] A. Ekinci, A. O. Akdemir and M. E. Ozdemir, Integral Inequalities for Different Kinds of Convexity via Classical Inequalities, Turk. J. Sci., 5, No. 3 (2020) 305-313.

[53] A. Ekinci and M. E. Ozdemir, Some New Integral Inequalities Via Riemann-Liouville Integral Operator, Appl. Comput. Math., 18, No. 3 (2019) 288-295.

[54] D. Nie, S. Rashid, A. O. Akdemir, D. Baleanu and J. Liu, On Some New Weighted Inequalities for Differentiable Exponentially Convex and Exponentially Quasi-Convex Functions with Applications, Mathematics, 7 , No. 8 (2019) 727.

[55] S. I. Butt, M. Nadeem and G. Farid, On Caputo Fractional Derivatives via Exponential s-Convex Functions, Turk. J. Sci. textbf5, No. 2 (2020) 140-146. 
[56] A. O. Akdemir, A. Ekinci and E. Set, Conformable Fractional Integrals And Related New Integral Inequalities, J Nonlinear Convex Anal. (2017)18, No. 4 (2017)661-674.

[57] M. E. Ozdemir, M. E. Latif and A. O. Akdemir, On Some Hadamard-Type Inequalities for Product of Two h-Convex Functions On the Coordinate, Turk. J. Sci. 1, No. 41 (2016) 41-58.

[58] E. Set, A. O. Akdemir and F. Demirci, Grüss Type Inequalities for Fractional Integral Operator Involving the Extended Generalized Mittag-Leffler Function, Appl. Comput. Math. 19, No. 3 (2019) 402-414.

[59] S. S. Zhou, S. Rashid, E. Set, A. G. Ahmad and Y.S. Hamed, On more general inequalities for weighted generalized proportional Hadamard fractional integral operator with applications, AIMS Math. 6, No. 9 (2021) 9154-9176. DOI: 10.3934/math.2021532.

[60] S. Rashid, K. T. Kubra, A. Rauf and Y. M. Chu and Y. S. Hamed, Hamed New numerical approach for timefractional partial di? erential equations arising in physical system involving natural decomposition method, Physica Scr. 96, No. 10 (2021). DOI: 10.1088/1402-4896/ac0bce

[61] S. Rashid, F. Jarad, H. Kalsoom and Y. M. Chu, More new results on integral inequalities for generalized K-fractional conformable integral operators, Discrete. Cont. Dyn. Sys.- Ser S, (2021). DOI: $10.3934 /$ dcdss. 2021063

[62] S. S. Zhou, S. Rashid, A. Rauf, Y. S. Hamed and K. M. Abualnaja, Efficient computations for weighted generalized proportional fractional operators with respect to a monotone function, AIMS Mathematics, $\mathbf{6}$, No. 8 (2021) DOI: 10.3934/math.2021465

[63] S. Rashid, S. Sultana F. Jarad and H. Jafari, Hamed Y S. More efficient estimates via h-discrete fractional calculus theory and applications, Chaos Solit. Fract, 147 (2021) 110981.

[64] H. Ge-Jile, S. Rashid, F. B. Farooq and S. Sultana, Some inequalities for a new class of convex functions with applications via local fractional integral, J. Fun. Spaces, 2021, No. 1 (2021). DOI: 10.1155/2021/6663971.

[65] S. Rashid, S. Parveen, H. Ahmad, and Y. M. Chu, New quantum integral inequalities for some new classes of generalized $\psi$-convex functions and their scope in physical systems, Open Phy. 19. No. 1 (2021) DOI: 10.1515/phys-2021-0001. 